Mon. Not. R. Astron. Soc. 000, 000-000 (0000) Printed 9 November $2018 \quad$ (MN LATEX style file v2.2)

\title{
Massive black hole binary plane reorientation in rotating stellar systems
}

\author{
Alessia Gualandris ${ }^{1 \star}$, Massimo Dotti ${ }^{2}$, and Alberto Sesana ${ }^{3}$ \\ ${ }^{1}$ Max-Planck Institut für Astrophysik, Karl-Schwarzschild-Str. 1, D-85741 Garching, Germany \\ ${ }^{2}$ Dipartimento di Fisica G. Occhialini, Università degli Studi di Milano Bicocca, Piazza della Scienza 3, 20126 Milano, Italy \\ ${ }^{3}$ Albert Einstein Institut, Am Mühlenberg 1, Golm, D-14476, Germany.
}

\begin{abstract}
We study the evolution of the orientation of the orbital plane of massive black hole binaries (BHBs) in rotating stellar systems in which the total angular momentum of the stellar cusp is misaligned with respect to that of the binary. We compare results from direct summation $N$-body simulations with predictions from a simple theoretical model. We find that the same encounters between cusp stars and the BHB that are responsible for the hardening and eccentricity evolution of the binary, lead to a reorientation of the binary orbital plane. In particular, binaries whose angular momentum is initially misaligned with respect to that of the stellar cusp tend to realign their orbital planes with the angular momentum of the cusp on a timescale of a few hardening times. This is due to angular momentum exchange between stars and the BHB during close encounters, and may have important implications for the relative orientation of host galaxies and radio jets.
\end{abstract}

Key words: black hole physics - methods: numerical - stellar dynamics

\section{INTRODUCTION}

In the standard $\Lambda \mathrm{CDM}$ cosmological scenario, massive black hole binaries (BHBs) are the natural outcome of mergers of massive galaxies (Begelman et al. 1980). The orbital decay of BHBs has been studied in detail under the assumption of purely stellar systems (e.g. Makino \& Funato 2004; Berczik et al. 2005; Baumgardt et al. 2006; Merritt et al. 2007; Sesana et al. 2007; Sesana 2010) as well as gaseous environments (e.g. Armitage \& Natarajan 2002, 2005; Escala et al. 2004, 2005; Dotti et al. 2006, 2007, 2009; Cuadra et al. 2009; Lodato et al. 2009; Roedig et al. 2011).

Most studies of the evolution of BHBs in stellar environments consider non rotating, spherically symmetric nuclei, despite the natural occurrence of rotation in merger remnants (e.g. Gualandris \& Merritt 2011). Simulations of MBHs in spherical stellar systems predict a stalling of the binary evolution at parsec scale separations, the "final parsec problem" (Milosavliević \& Merritt 2003), which may or may not be avoided in the presence of gas dissipation. A BHB can, however, reach coalescence even in a completely gas poor environment, if embedded in a non-spherically symmetric stellar distribution. Purely dissipationless simulations of non-spherical galaxy models (Berczik et al. 2006; Berentzen et al. 2009) or galaxy mergers (Khan et al. 2011;

* E-mail: alessiag@mpa-garching.mpg.de
Preto et al. 2011; Gualandris \& Merritt 2011) find continuing hardening of the binary down to separations where energy loss due to emission of gravity waves becomes efficient. Simulations by Gualandris \& Merritt (2011) show that efficient hardening is achieved even in systems close to axisymmetry. This departure from spherical symmetry is due to the rotation introduced by the merger, which results in an enhanced rate of stars interacting with the binary.

A comprehensive and systematic study of the evolution of BHBs in rotating stellar cusps is still missing. Sesana et al. (2011) (hereafter paper I) studied the evolution of the eccentricity of unequal mass BHBs in rotating systems by means of an hybrid analytical/3-body scattering formalism as well as full $N$-body simulations. They found a strong dependence of the eccentricity evolution of the binary on the degree of co-rotation in the stellar cusp, with binaries increasing their eccentricity in cusps containing a significant fraction of counter-rotating stars. This is relevant to the case of galactic minor mergers, in which counter-rotating systems can be produced as a result of rotation in the larger galaxy, thereby forming very eccentric binaries.

Another important and potentially observable parameter is the orientation of the binary orbital plane. Its evolution has been studied in spherical and isotropic systems (Merritt 2002; Gualandris \& Merritt 2007). Due to the lack of any preferential direction in these systems, the orbital plane of the binary can only undergo a random walk, result- 
ing in small changes in the orbital plane on long timescales. Here we study the evolution of the orbital plane of BHBs in rotating stellar systems, where, as will be discussed, a significantly larger reorientation is expected.

The letter is organized as follows. In section 2 we describe the evolution of the binary orbital plane in spherically symmetric models. In section 3 we describe the case of rotating models, comparing results from $N$-body simulations with a simple analytical model. Final remarks are presented in Section 4 .

\section{NON ROTATING CUSPS}

The orientation of the orbital plane of BHBs embedded in non rotating, isotropic stellar nuclei has been studied for the first time by Merritt (2002). In such systems, repeated encounters with passing stars cause the orientation of the binary to undergo a random walk (a "rotational Brownian motion", in Merritt (2002)). From simple analytical arguments, the expected change in orientation on one hardening time $\mathrm{T}_{\mathrm{h}}=|\mathrm{a} / \dot{\mathrm{a}}|$ of the binary (which is of the order of 120 initial binary orbital periods) is (Gualandris \& Merritt 2007)

$$
\Delta \theta \propto q^{-1 / 2}\left(\frac{m_{*}}{M_{\mathrm{BHB}}}\right)^{1 / 2}\left(1-e^{2}\right)^{-1 / 2}
$$

where $q=M_{2} / M_{1}$ is the binary mass ratio, $M_{\mathrm{BHB}}$ the total mass, and $e$ the orbital eccentricity. Numerical scattering experiments have constrained the normalization in Eq. 1 only for circular binaries. Neither the dependence on the eccentricity nor on the mass ratio has been tested numerically. In the case of a binary with $M_{\mathrm{BHB}}=10^{6} m_{*}$ and $q=10^{-3}$, the expected reorientation is $\Delta \theta \approx 9^{\circ}$.

Here we check the validity of Eq. 1 by performing $N$ body simulations of binaries with different eccentricities (in the range $0-0.99$ ) embedded in non-rotating stellar cusps. We use the direct summation $N$-body code $\phi$ GRAPE (Harfst et al. 2007) in combination with the Sapporo library (Gaburov et al. 2009) to accelerate the computations on GPU hardware. In our integrations we adopted $M_{1}=$ $10^{6} \mathrm{M}_{\odot}$ and $q=1 / 81$ and set the initial semi-major axis to $a_{i}=0.06 \mathrm{pc}$. The MBHs are embedded into a stellar cusp following a Bahcall-Wolf $\rho(r) \sim r^{-7 / 4}$ density profile at distances smaller than $1 \mathrm{pc}$, with total mass $M_{c} \sim 2.5 \times 10^{5} \mathrm{M}_{\odot}$ and a mass enclosed in the binary orbit equal to $2 M_{2}$. The cusp is modelled with $N=32 \mathrm{k}$ equal-mass particles, resulting in a black hole to star mass ratio of $m_{*} / M_{1}=7.5 \times 10^{-6}$. Fig 1 shows the degree of re-alignment of the binary plane as a function of the initial eccentricity. The dashed line indicates the dependence expected from the analytical model, while the points show the results of our $N$-body integrations. The agreement is remarkable.

For a given binary, the re-orientation in a non-rotating system does not depend only on the mass density and velocity field of the stars, but also on the mass ratio between the binary and a single star in the cusp. 1 . A binary embed-

1 Note that this ratio is properly defined only for a single mass stellar cusp. In the presence of a mass spectrum, the mean mass of the interacting stars is a good proxy for $m_{*}$.

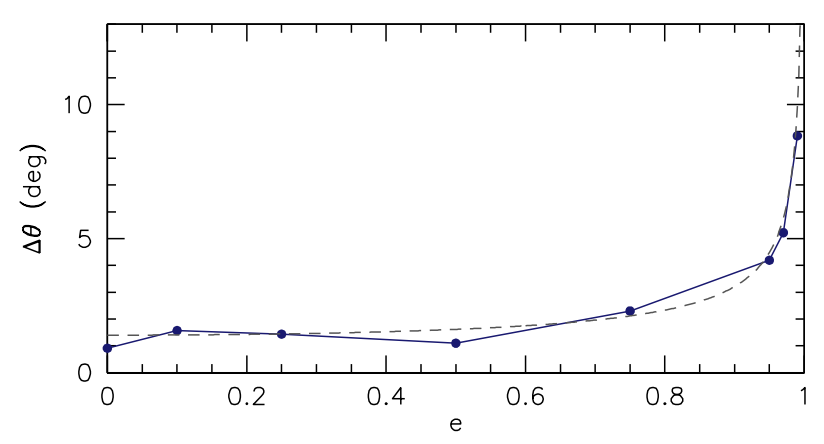

Figure 1. Change over one hardening time in the orientation of the angular momentum of the binary, as a function of the initial eccentricity of the BHB. The dashed line represents the theoretical dependence given in Eq. 1 normalized to match the data at $e=0.25$. The amplitude of the reorientation in our runs is however comparable to the analytical estimates in Merritt (2002).

ded in a stellar cusp would, on average, experience a greater realignment as a result of few encounters with massive stars than as a result of many encounters with low-mass stars. This feature is typical of any random walk process, in which there is no preferential direction for the change of the binary plane in a single encounter and changes due to different encounters tend to cancel each other out. The same random walk behaviour is observable in rotating cusps, if the angular momentum of the binary is aligned with that of the cusp (Amaro-Seoane et al. 2010). This behaviour is illustrated in the lower panel of Fig 2 which shows the dependence of the change in $\theta$ on the number of particles used to model the cusp, i.e., on the $m_{*} / M_{\mathrm{BHB}}$ mass ratio. The agreement between the numerical results (full circles) and Eq. 1 (solid line) is outstanding.

\section{ROTATING CUSPS}

Here we study in detail the re-orientation of a massive BHB embedded in a cusp with net rotation. To this aim, we perform high accuracy $N$-body simulations similar to those presented in the previous section, the only difference being the degree of rotation that we enforce in the cusp. This is obtained, as in Paper I, by reversing the sign of all velocity components for a random subset of cusp stars, at the time where the BHB is added. In particular, we generated models with a fraction $\mathcal{F}=0.875$ of co-rotating stars and varied the initial angle $\theta_{0}$ between the total angular momentum of the cusp and the angular momentum of the binary. We considered cases with $\theta_{0}=0^{\circ}, 30^{\circ}, 60^{\circ}, 90^{\circ}, 120^{\circ}, 150^{\circ}$.

The evolution of $\theta$ as a function of time is shown in Fig. 3 (solid lines) for all runs. Binaries whose angular momentum is initially mis-aligned with respect to that of the cusp tend to realign it on a time-scale of a few hardening times. The re-alignment can be quite significant, with an average change in $\theta$ of the order of $50 \%$ of the initial value, and up to $70 \%$ in some cases. For example, the case with $\theta_{0}=150^{\circ}$ reaches a final value of $\theta \sim 45^{\circ}$, with a change of more than $100^{\circ}$ over one hardening time. This is about 100 times larger than the reorientation measured for a similar isotropic model (see Fig. 1). As will be discussed below, such large reorientation of the orbital plane may have 


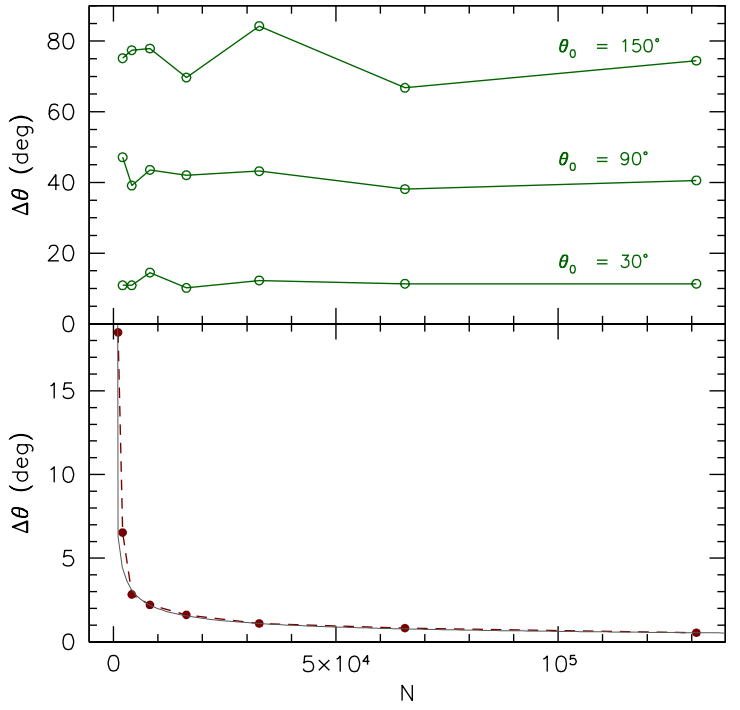

Figure 2. Change in the binary plane orientation per hardening time versus number of particles in the cusp (in the range $N=2 \mathrm{k}$ to $N=128 \mathrm{k}$ ) or, equivalently, ratio between star mass and $\mathrm{MBH}$ mass. In all the runs $e=0.5$. The lower panel refers to isotropic models, while the upper panel refers to rotating models with $\mathcal{F}=$ 0.875 and an initial angle between the binary angular momentum and that of the stellar cusp $\theta_{0}=30^{\circ}, 90^{\circ}, 150^{\circ}$.



Figure 3. Evolution of the angle $\theta$ between the angular momentum vector of the binary and that of the stellar cusp. Time is expressed in units of the initial binary orbital period (bottom) and of the hardening time (top). Different solid lines are for models with different initial values of $\theta$, from $\theta_{0}=0^{\circ}$ to $\theta_{0}=150^{\circ}$. Dotted lines represent the predictions from the theoretical model while dashed lines indicate the predictions modified to take into account the finite reservoir of angular momentum in the stellar cusp.

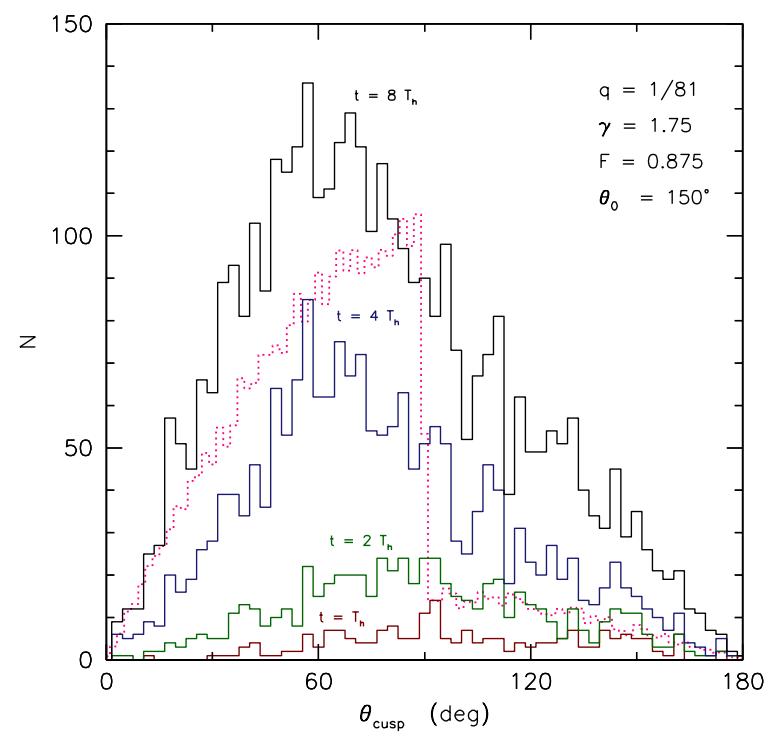

Figure 4. Solid lines: Distribution of the angle $\theta_{\text {cusp }}$ between the angular momentum vector of a single star and the initial angular momentum vector of the cusp, for the run with $\theta_{0}=150^{\circ}$. Only unbound stars are considered. The different curves refer to different times during the evolution, from $1 \mathrm{~T}_{\mathrm{h}}$ to $8 \mathrm{~T}_{\mathrm{h}}$. Stars are ejected roughly isotropically from early times. Dotted line: Initial distribution of $\theta_{\text {cusp }}$ for all cusp stars, normalized by a factor of 10 , for an easy comparison.

observational consequences. However, we note that the realignment is not complete but stops at a finite value of $\theta$.

The evolution of $\theta$ can be explained in terms of a simple analytical model. Let $\boldsymbol{l}_{B}=\boldsymbol{L}_{B} / \mu$ be the angular momentum per unit mass of the binary, with $\mu=M_{1} M_{2} /\left(M_{1}+M_{2}\right)$ the reduced mass, and $\boldsymbol{l}_{\star}=\boldsymbol{L}_{\star} / m_{\star}$ the angular momentum per unit mass of a star interacting with the binary. Conservation of angular momentum during an encounter (assuming $m_{\star} \ll$ $\left.M_{1}+M_{2}\right)$ gives

$$
\Delta \boldsymbol{l}_{B}=\frac{m_{\star}}{\mu} \Delta \boldsymbol{l}_{\star} .
$$

Considering that, once normalized per unit mass, the average change per encounter in the stellar angular momentum is of the order of the binary angular momentum (Merritt 2002) $)^{2}$, each interaction contributes, on average, a factor

$$
<\Delta \boldsymbol{l}_{B}>\sim \frac{m_{\star}}{\mu} \boldsymbol{l}_{B}
$$

to the binary angular momentum. The rate at which $\boldsymbol{l}_{B}$ varies with time is

$$
\frac{\mathrm{d} \boldsymbol{l}_{B}}{\mathrm{~d} t}=\frac{\mathrm{d} n_{\mathrm{enc}}}{\mathrm{d} t}<\Delta \boldsymbol{l}_{B}>,
$$

where $\mathrm{d} n_{\mathrm{enc}} / \mathrm{d} t$ is the rate of binary/star interactions. The

2 This allows us to ignore the evolution of the magnitude of the binary angular momentum due to hardening when modelling the evolution of the plane orientation. The change in $\theta$ would be the same even if we considered the decay of the binary orbit. 


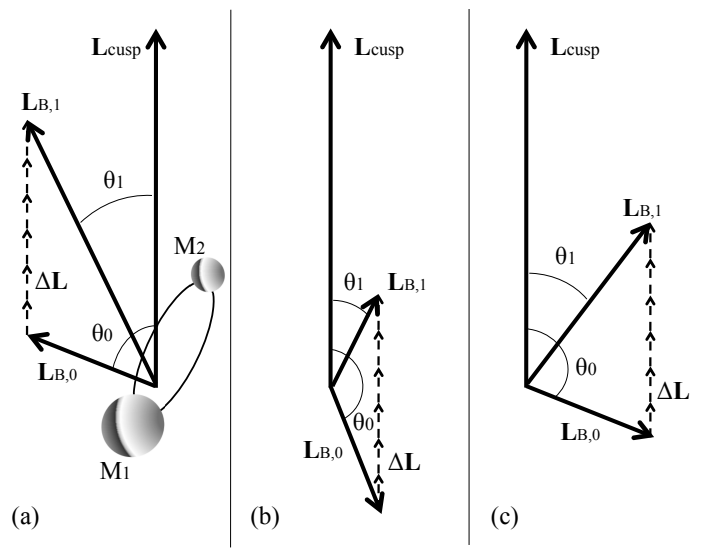

Figure 5. Sketch of the model explaining the re-alignment of the binary plane. $\boldsymbol{L}_{\mathrm{B}, 0}$ is the initial angular momentum of the binary (perpendicular to the orbital plane, shown for clarity in the left panel). $\boldsymbol{L}_{\mathrm{B}, 1}$ is the angular momentum of the binary after realignment. $\Delta \boldsymbol{L}$ is the amount of angular momentum transferred at any interaction with a single star (dashed line). Note that we assume $\Delta \boldsymbol{L}$ parallel to the total angular momentum $\boldsymbol{L}_{\text {cusp }}$ of the cusp. $\theta_{0}$ and $\theta_{1}$ indicate, respectively, the initial and final angles between $\boldsymbol{L}_{\mathrm{B}}$ and $\boldsymbol{L}_{\text {cusp }}$. Panel (a) shows a case with $\boldsymbol{L}_{\mathrm{B}, 0}$ partially aligned with $\boldsymbol{L}_{\text {cusp }}\left(\theta_{0}<90^{\circ}\right)$. Panels (b) and (c) refer to the opposite case $\left(\theta_{0}>90^{\circ}\right)$. Vectors are not on a physical scale.

rate can be estimated as

$$
\begin{aligned}
\frac{\mathrm{d} n_{\mathrm{enc}}}{\mathrm{d} t} & =\pi n_{*} R_{\mathrm{i}, 2}^{2} v_{\mathrm{rel}} \\
& \approx \pi n_{*}\left(\frac{G M_{2}}{v_{\mathrm{rel}}^{2}}\right)^{2} v_{\mathrm{rel}} \\
& \approx \pi n_{*}\left(\frac{M_{2}}{M_{1}}\right)^{2} \sqrt{G M_{1} a^{3}},
\end{aligned}
$$

where $n_{*}$ is the number density of stars in the cusp, $R_{\mathrm{i}, 2}$ is the influence radius of the secondary hole, and $v_{\text {rel }}$ is the mean relative velocity between the stars and $M_{2}$. Note that we have implicitly assumed that: i) only stars efficiently interacting with the secondary modify the angular momentum of the binary; ii) the dynamics outside the influence radius of the secondary is completely dominated by the primary.

In order to apply Eqs.25. we need to specify the average direction of $\left\langle\Delta \boldsymbol{l}_{\mathrm{B}}\right\rangle$. This is opposite to the direction of the variation of the angular momentum of the star during the interaction. In a reference frame in which the $z$ direction is aligned with the total angular momentum of the cusp, the average $x$ and $y$ components of the angular momentum of the stars before an encounter are zero. In this simple model we assume that the final angular momentum of a star that experienced an interaction is isotropically distributed. This is justified by the observation that, at any time, stars after an interaction with the binary are much more isotropically distributed than before, as shown in Fig. 4 for the population of unbound stars. As a consequence, the variation $<\Delta \boldsymbol{l}_{*}>=<\boldsymbol{l}_{*, 1}-\boldsymbol{l}_{*, 0}>$ points in the direction opposite to the angular momentum of the cusp $\boldsymbol{l}_{\text {cusp }}$, and $\left\langle\Delta \boldsymbol{l}_{B}\right\rangle$, averaged over repeated encounters, points in the $z$ direction, parallel to $\boldsymbol{l}_{\text {cusp }}$. The fast alignment that we observe in the $N$-body simulations is a direct consequence of such prefer- ential direction of $\Delta \boldsymbol{l}_{B}$. Fig. 5 shows a schematic view of our model, for different initial orientations of the binary.

The evolution of $\theta$ predicted by our model is shown with dotted lines in Fig. 3 Naturally, the case with $\boldsymbol{l}_{B}$ aligned with $\boldsymbol{l}_{\text {cusp }}$ does not show any evolution. Despite the simplifying assumptions made, the agreement between the initial evolution of $\theta$ in the runs and in the model is very good.

The predictions of the model and the results of the $N$-body runs disagree at late times. In the simulations the binary stops re-orienting well before perfect alignment is reached, while the model predicts $\theta \rightarrow 0$ asymptotically. This difference can be explained simply in terms of a depletion of stars in the centre of the cusp. As in many previous studies (e.g. Milosavljević \& Merritt 2001; Milosavliević et al. 2002; Merritt 2006; Merritt et al. 2007; Gualandris \& Merritt 2011), we observe the formation of a central core due to slingshot ejections of stars. After a core has been excavated in the centre, the rate of encounters drastically drops and the evolution of the BHB's orbital plane halts. This translates into a maximum angular momentum $l_{\text {max }}$ that can be transferred from the stars to the binary . Since in our model we assumed the transferred angular momentum to depend on the properties of the cusp and not on those of the binary (the stars are ejected isotropically), $l_{\max }$ must be the same in every run. We computed the value of $l_{\max }$ that needs to be transferred to the binary to match exactly the evolution of the $\theta_{0}=120^{\circ} \mathrm{run}$, and we checked if such a limiting angular momentum can account for the other runs' behaviour. The results of this test are shown in Fig. 3 , with dashed lines. The agreement with the $N$-body results is greatly improved by the introduction of a maximum angular momentum that can be transferred to the binary.

As a final note, we emphasize that the re-alignment in rotating cusps does not depend on the $m_{*} / M_{\mathrm{BHB}}$ ratio, as opposed to the non-rotating case. Since the rate of encounters under every assumption depends on the number density of stars, and the amount of angular momentum transferred per encounter is proportional to $m_{*}, \mathrm{~d} l_{\mathrm{B}} / \mathrm{d} t$ depends on the mass density $\rho$ of the cusp, but does not show any other dependence on $m_{*} / M_{\mathrm{BHB}}$. This is a consequence of the fact that the alignment in each encounter has a preferential direction (i.e. towards the angular momentum of the cusp). The upper panel in Fig. 2 shows the alignment $\Delta \theta$ experienced over an hardening time by binaries in rotating cusps, as a function of the number of stars in the cusp. Different lines are for different initial angles between $\boldsymbol{l}_{\mathrm{B}}$ and $\boldsymbol{l}_{\text {cusp }}$. While in the non-rotating case the ratio between the re-alignments in the lowest and highest mass resolution runs is $\sim 30$, in this case the re-alignment shows only statistical fluctuations, with no systematic trends.

\section{CONCLUSIONS}

We studied the evolution of the orientation of the orbital plane of unequal mass BHBs in isotropic and rotating stellar systems. The evolution in isotropic systems is characterized by a random walk on long time-scales, as predicted by Merritt (2002). The evolution in systems in which a fraction of stars is counter-rotating with respect to the binary was briefly discussed in Sesana et al. (2011). If the fraction of counter-rotating stars is small, the change in the orbital 
Massive black hole binary plane reorientation

plane is consistent with the change expected for isotropic cusps. If, on the other hand, a large fraction of stars is counter-rotating, the plane of the binary evolves considerably and the binary angular momentum even reverses in the case of a fully counter-rotating cusp.

Here, we investigated for the first time the evolution of the binary plane in rotating systems in which the binary angular momentum is initially misaligned with respect to that of the stellar cusp. We find that mis-aligned binaries in rotating cusps tend to align their angular momentum with that of the cusp on a time-scale of a few hardening times. The alignment process is linear with time, hence, unlike the random walk observed in fully isotropic systems, does not depend on the mass ratio between the MBHs and the stars.

The realignment of the binary plane that we observe in the simulations may have significant implications. The direction of the spin axis of the single $\mathrm{MBH}$ that results from the merger of the holes due to emission of gravitational waves is affected by the orientation of the binary plane before coalescence. The spin axis, in turn, determines the orientation of the accretion disk around the remnant black hole via the Bardeen-Peterson effect (Bardeen \& Petterson 1975) and, in radio-loud AGNs, the direction of the radio jet.

After the coalescence of a $\mathrm{BHB}$, the spin of the remnant $\boldsymbol{j}_{\mathrm{r}}$ is not necessarily aligned with the spins of the two progenitors, $\boldsymbol{j}_{1}$ and $\boldsymbol{j}_{2}$. If one of the two MBHs in the binary is radio-loud (and the remnant stays radio-loud), the change in spin direction would result in a reorientation of the relativistic jet 3 (Merritt \& Ekers 2002). The orientation of the orbital plane of the binary has implications for the direction of $\boldsymbol{j}_{\mathrm{r}}$. The interaction with a rotating cusp tends to force the binary to co-rotate with the central cusp. At coalescence, the binary orbital angular momentum contribution to $\boldsymbol{j}_{\mathrm{r}}$ will result in a spin (and possibly a jet) preferentially aligned with the cusp angular momentum.

We do not expect full alignment, however, because: (i) the alignment we find in the simulations is not complete, and could halt in the absence of further refilling of stars or in the presence of isotropic refilling, leaving misalignment angles of up to $60^{\circ}$, (ii) in a very unequal-mass binary (e.g. $q=1 / 81$, like the one we study), $\boldsymbol{j}_{\mathrm{r}}$ depends strongly on $\boldsymbol{j}_{1}$. Using the results of Rezzolla et al. (2008), if $j_{1} \approx 1$, the angle between $\boldsymbol{j}_{1}$ and $\boldsymbol{j}_{\mathrm{r}}$ is of only a few degrees, while $j_{1} \approx 0.1$ results in an angle of $\gtrsim 20^{\circ}$.

Such a preferential alignment is in fact observed. In weak radio-loud AGNs, Browne \& Battye (2010) found a tendency for the axis of the radio emission to align with the minor axis of the starlight of the host, an oblate rotationally supported elliptical. By contrast, they found no preferred radio-optical alignment among the radio-louder objects, (see also Saripalli \& Subrahmanyan 2009), possibly hosted in triaxial non-rotating ellipticals (e.g. Kormendy et al. 2009).

3 This is valid in gas poor mergers. In gas rich environments, gas accretion causes the spins of the MBHs to align with the angular momentum of the binary (Bogdanović et al. 2007; Dotti et al. 2010; Volonteri et al. 2010; Kesden et al. 2010), preventing any drastic change in the spins direction at coalescence.

\section{ACKNOWLEDGMENTS}

We thank Pau Amaro-Seoane and David Merritt for interesting discussions and the anonymous referee for useful comments on the manuscript.

\section{REFERENCES}

Amaro-Seoane P., Eichhorn C., Porter E. K., Spurzem R., 2010, MNRAS, 401, 2268

Armitage P. J., Natarajan P., 2002, ApJL, 567, L9

Armitage P. J., Natarajan P., 2005, ApJ, 634, 921

Bardeen J. M., Petterson J. A., 1975, ApJL, 195, L65+

Baumgardt H., Gualandris A., Portegies Zwart S., 2006, MNRAS, 372, 174

Begelman M. C., Blandford R. D., Rees M. J., 1980, Nature, 287, 307

Berczik P., Merritt D., Spurzem R., 2005, ApJ, 633, 680

Berczik P., Merritt D., Spurzem R., Bischof H.-P., 2006, ApJL, 642, L21

Berentzen I., Preto M., Berczik P., Merritt D., Spurzem R., 2009, ApJ, 695, 455

Bogdanović T., Reynolds C. S., Miller M. C., 2007, ApJL, 661, L147

Browne I. W. A., Battye R. A., 2010, in L. Maraschi, G. Ghisellini, R. Della Ceca, \& F. Tavecchio ed., Accretion and Ejection in AGN: a Global View Vol. 427 of Astronomical Society of the Pacific Conference Series, A Dichotomy in Radio Jet Orientations in Elliptical Galaxies. pp 365-+

Cuadra J., Armitage P. J., Alexander R. D., Begelman M. C., 2009, MNRAS, 393, 1423

Dotti M., Colpi M., Haardt F., 2006, MNRAS, 367, 103

Dotti M., Colpi M., Haardt F., Mayer L., 2007, MNRAS, 379, 956

Dotti M., Ruszkowski M., Paredi L., Colpi M., Volonteri M., Haardt F., 2009, MNRAS, 396, 1640

Dotti M., Volonteri M., Perego A., Colpi M., Ruszkowski M., Haardt F., 2010, MNRAS, 402, 682

Escala A., Larson R. B., Coppi P. S., Mardones D., 2004, ApJ, 607, 765

Escala A., Larson R. B., Coppi P. S., Mardones D., 2005, ApJ, 630, 152

Gaburov E., Harfst S., Portegies Zwart S., 2009, New A, 14,630

Gualandris A., Merritt D., 2007, ArXiv e-prints

Gualandris A., Merritt D., 2011, ArXiv e-prints

Harfst S., Gualandris A., Merritt D., Spurzem R., Portegies

Zwart S., Berczik P., 2007, New Astronomy, 12, 357

Kesden M., Sperhake U., Berti E., 2010, ApJ, 715, 1006

Khan F. M., Just A., Merritt D., 2011, ApJ, 732, 89

Kormendy J., Fisher D. B., Cornell M. E., Bender R., 2009, ApJS, 182, 216

Lodato G., Nayakshin S., King A. R., Pringle J. E., 2009, MNRAS, 398, 1392

Makino J., Funato Y., 2004, ApJ, 602, 93

Merritt D., 2002, ApJ, 568, 998

Merritt D., 2006, ApJ, 648, 976

Merritt D., Ekers R. D., 2002, Science, 297, 1310

Merritt D., Mikkola S., Szell A., 2007, ApJ, 671, 53

Milosavljević M., Merritt D., 2001, ApJ, 563, 34 
Milosavljević M., Merritt D., 2003, ApJ, 596, 860

Milosavljević M., Merritt D., Rest A., van den Bosch F. C., 2002, MNRAS, 331, L51

Preto M., Berentzen I., Berczik P., Spurzem R., 2011, ApJL, 732, L26+

Rezzolla L., Barausse E., Dorband E. N., Pollney D., Reisswig C., Seiler J., Husa S., 2008, Phys. Rev. D, 78, 044002 Roedig C., Dotti M., Sesana A., Cuadra J., Colpi M., 2011, MNRAS, pp 979-+

Saripalli L., Subrahmanyan R., 2009, ApJ, 695, 156

Sesana A., 2010, ApJ, 719, 851

Sesana A., Gualandris A., Dotti M., 2011, MNRAS, 415, L35

Sesana A., Haardt F., Madau P., 2007, ApJ, 660, 546

Volonteri M., Gültekin K., Dotti M., 2010, MNRAS, 404, 2143 